\title{
Supporting Information \\ Collective nanoparticle dynamics associated with bridging network formation in model polymer nanocomposites
}

\author{
Benjamin M. Yavittt ${ }^{1}$, Daniel Salatto ${ }^{1}$, Yuxing Zhou ${ }^{2,3}$, Zhixing Huang ${ }^{1}$, Maya Endoh ${ }^{1}$, Lutz \\ Wiegart ${ }^{4}$, Vera Bocharova ${ }^{5,6}$, Alexander E. Ribbe ${ }^{7}$, Alexei P. Sokolov ${ }^{5,6, *}$, Kenneth S. \\ Schweizer ${ }^{2,3,8, *}$, Tadanori Koga ${ }^{1,9, *}$
}

${ }^{1}$ Department of Materials Science and Chemical Engineering, Stony Brook University, Stony Brook, NY 11794-2275, United States

${ }^{2}$ Department of Materials Science and Engineering, University of Illinois, Urbana, IL 61801, United States

${ }^{3}$ Materials Research Laboratory, University of Illinois, Urbana, IL 61801, United States

${ }^{4}$ National Synchrotron Light Source II, Brookhaven National Laboratory, Upton, NY, 11793, United States

${ }^{5}$ Chemical Sciences Division, Oak Ridge National Laboratory, Oak Ridge, TN 37831, United States

${ }^{6}$ Department of Chemistry, University of Tennessee Knoxville, University of Tennessee, Knoxville, TN 37996, United States

${ }^{7}$ Department of Polymer Science and Engineering, University of Massachusetts Amherst, Amherst, MA 01003, United States

${ }^{8}$ Department of Chemistry, University of Illinois, Urbana, IL 61801, United States

${ }^{9}$ Department of Chemistry, Stony Brook University, Stony Brook, NY, 11794-3400, United States

*Corresponding Authors 


\section{Additional Experimental Results and Characterizations.}

(a)

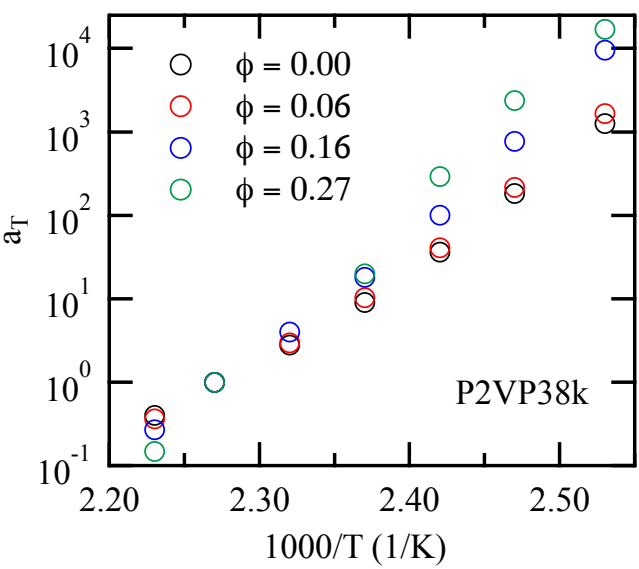

(c)

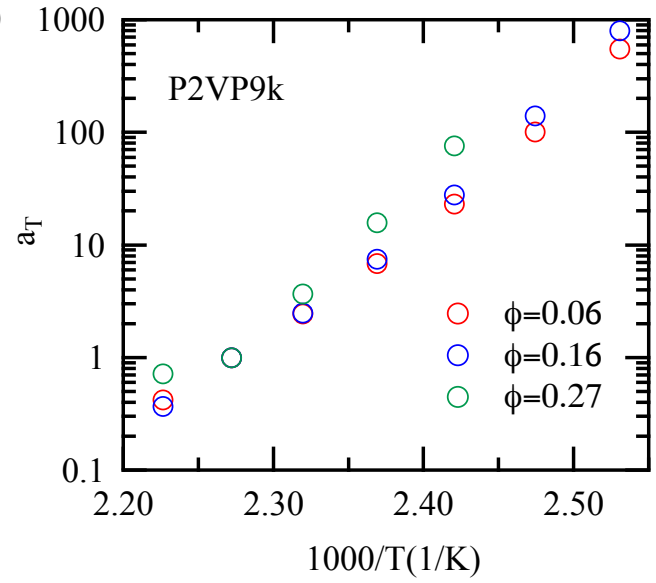

(b)

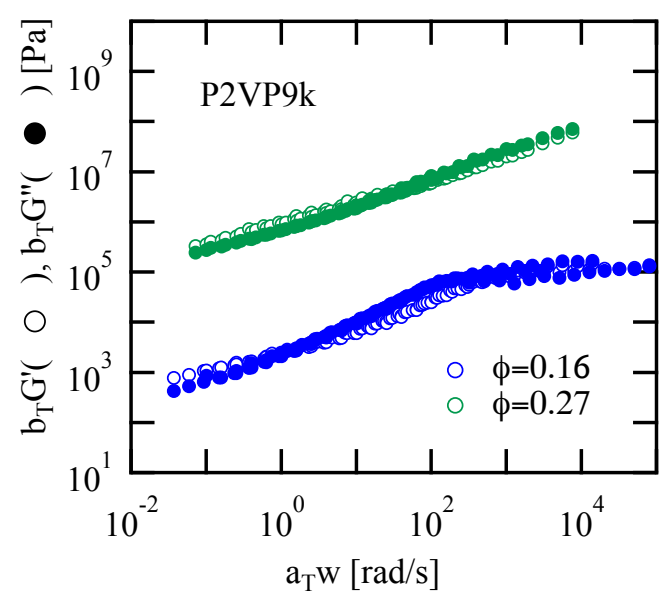

(d)

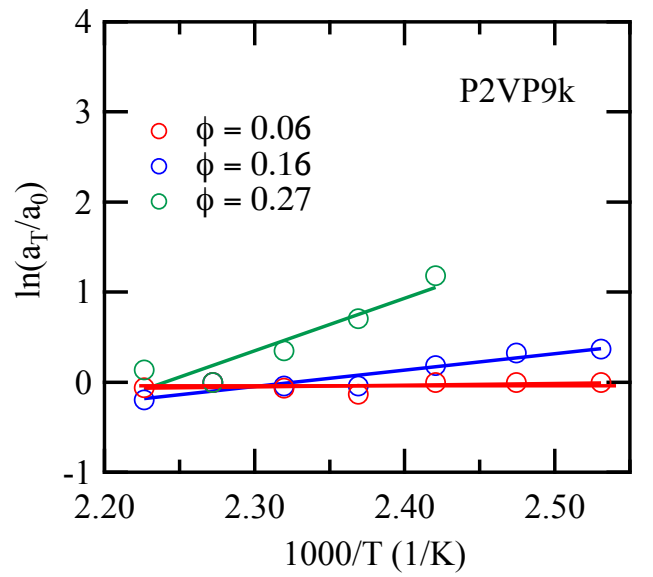

Figure S1. (a) Time-temperature shift factors $\left(a_{\mathrm{T}}\right)$ for the P2VP38k series and the neat polymer used in construction of dynamic master curves. The reference temperature $\left(T_{\text {ref }}\right)$ was set to $167^{\circ} \mathrm{C}$. (b) Dynamic master curves $G^{\prime}(\omega), G^{\prime}$ ' $(\omega)$ for the P2VP9k series generated by shifting small amplitude frequency sweeps according to time-temperature superposition $\left(T_{\text {ref }}=167^{\circ} \mathrm{C}\right)$. The dynamic master curves $G^{\prime}(\omega)$ and $G^{\prime \prime}(\omega)$ for $\phi=0.27$ are vertically shifted by an additional shift factor $b_{\mathrm{T}}$ for clarity: $b_{\mathrm{T}}=10$. The master curves $G^{\prime}(\omega)$ and $G^{\prime \prime}(\omega)$ and the time-temperature shift factors for the $\phi=0.06$ sample are indistinguishable from those for the neat P2VP9k (data not shown). (c) Time-temperature shift factors for the P2VP9k series used in construction of dynamic master curves. (d) Shift factor $\left(a_{\mathrm{T}}\right)$ for the P2VP9k series normalized by the shift factor for the neat P2VP9k melt $\left(a_{0}\right)$ as a function of inverse temperature. The solid lines show the best-fits of the Arrhenius temperature dependence for the PNCs using Eq. (1) in the main text. 


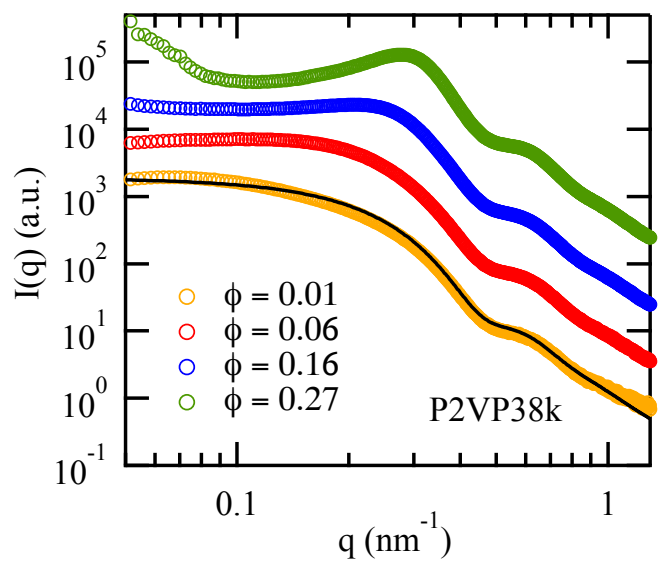

Figure S2. SAXS intensity profiles $I(q)$ (vertically shifted for clarity) for the P2VP38k series at $180{ }^{\circ} \mathrm{C}$. The solid line corresponds to the best-fit to the data at $\phi=0.01$ on the basis of a polydisperse core-shell particle model, motivated by previous results. ${ }^{1,2}$ It should be noted that the shell corresponds to the socalled "interfacial layer (IL)" 3 wich has a density ${ }^{1,2}$ different from that of the bulk. In addition, it has been reported that the thickness of the IL is $M_{\mathrm{w}}$ independent, ${ }^{4}$ in contrast to the BPL. ${ }^{3}$ Using the P2VP38K sample at $\phi=0.01$, the thickness of the IL was determined to be about $3 \mathrm{~nm}$, while the density is slightly higher than that of the bulk polymer by approximately $5 \%$. The details of the SAXS analysis have been described elsewhere. ${ }^{5}$ We also found no significant temperature dependence of $I(q)$ in the studied temperature range regardless of NP loadings. 

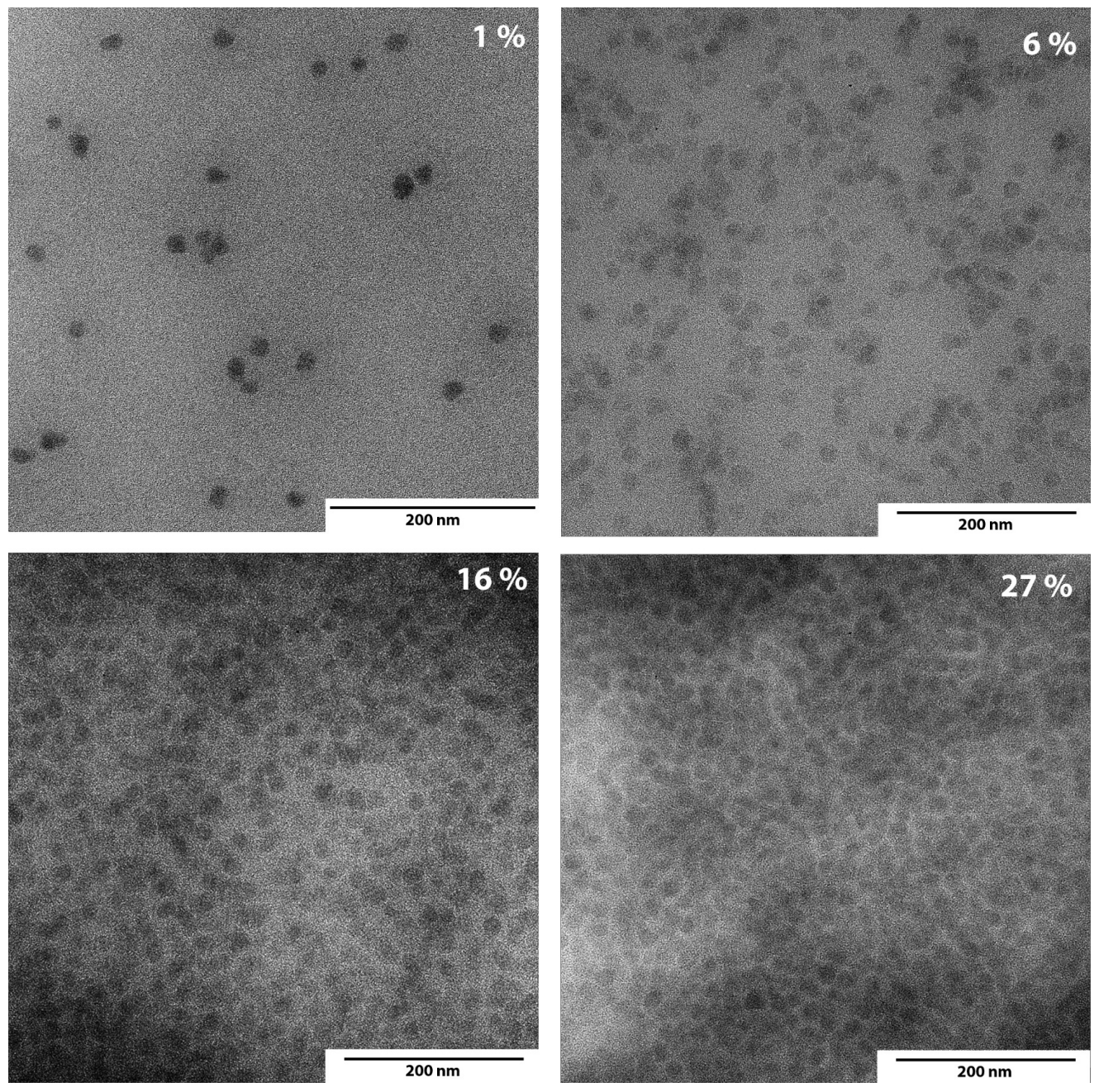

Figure S3. TEM images of the P2VP 38k series. As noted in the main text, the formation of local clusters occurs at $6 \%$, as theoretically expected. ${ }^{5}$ 


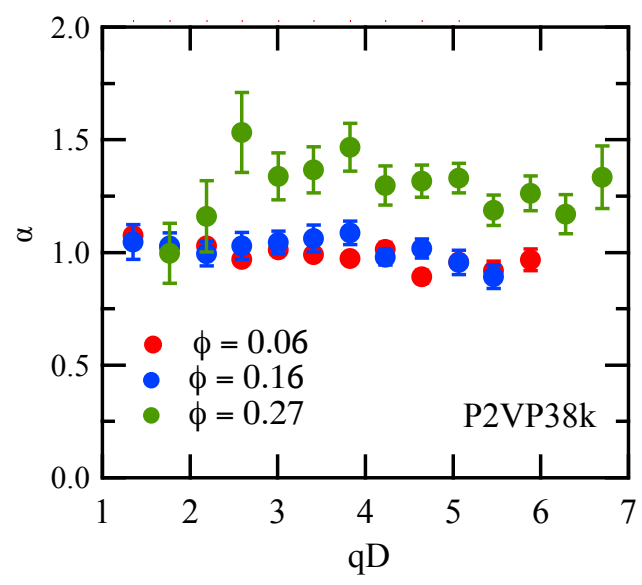

Figure S4. $q D$ dependence of the parameter $\alpha$ in Eq. (2) in the main text for the P2VP38k series at 180 ${ }^{\circ} \mathrm{C}$.

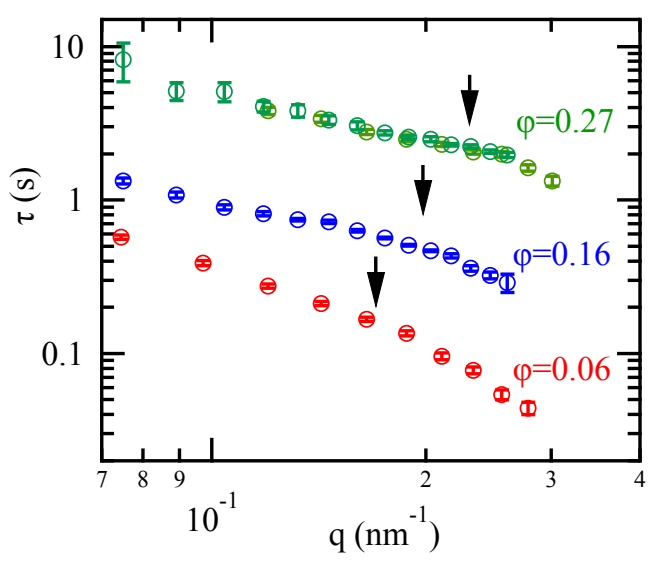

Figure S5. Relaxation time $\tau v s . q$ for the P2VP9k series at $180{ }^{\circ} \mathrm{C}$. The arrows correspond to the $q=q^{*}$ positions. 


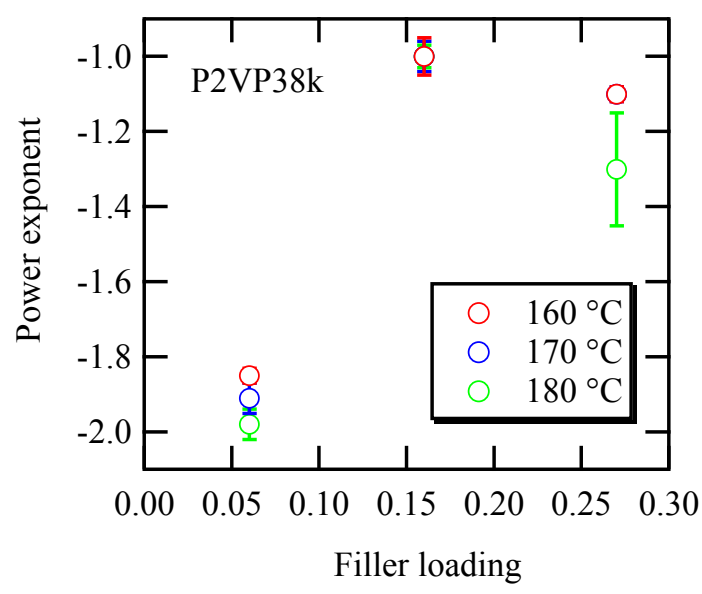

Figure S6. Local effective power exponent $v s$. filler loading for the P2VP38k series. The exponent ( $m$, i.e., $\tau \sim q^{m}$ ) was determined from the best-fit to the data (shown in Figure 3b) at $q<0.2 \mathrm{~nm}^{-1}$. It should be noted that the same trend of $m$ against filler loading is evidenced for the P2VP9k series, as shown in Figure 4.

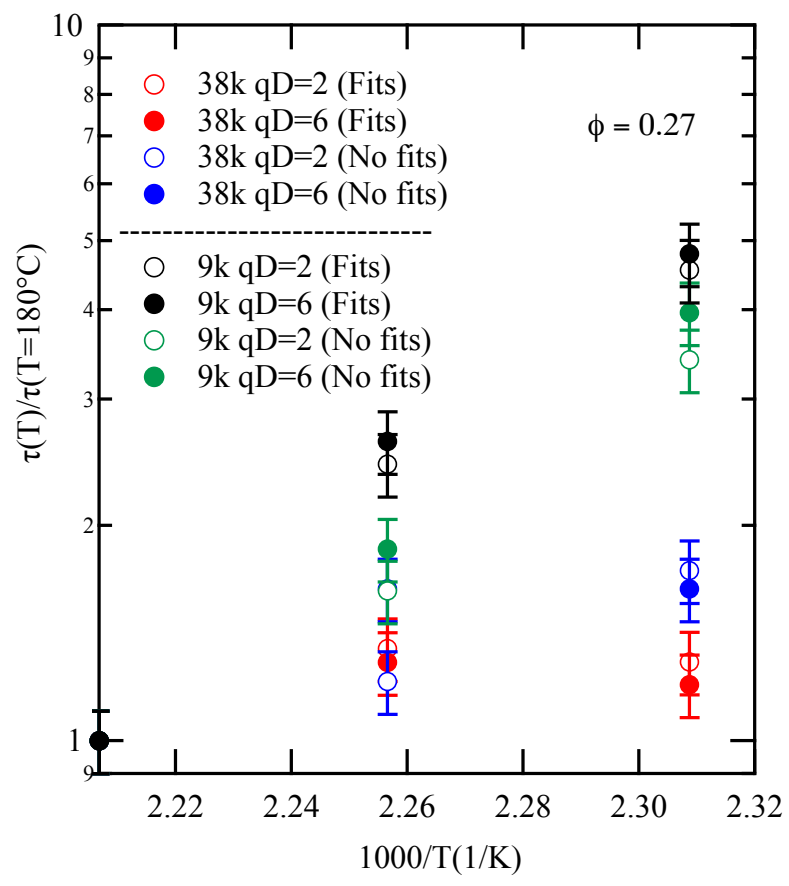

Figure S7. Temperature dependence of the normalized relaxation times at $q D=2$ and $q D=6$ for the P2VP9k and P2VP 38k series. As described in the main text, the observed $g_{2}$ functions can be reasonably fit with either a simple exponential function $(\alpha=1)$ for the $\phi=0.06$ and 0.16 samples or a compressed exponential function $(\alpha>1)$ for the $\phi=0.27$ samples. Hence, the unusual temperature dependence might reflect the method of $\tau$ extraction from the observed $g_{2}$ functions. To test this, we calculated $\tau$ by defining the delay time necessary for $g_{2}$ to decay to $1 / e$ of its initial value. The results show that the magnitude of $\tau$ extracted without fitting to KWW (represented as "No fits") is slightly different from the ones extracted by the fits using the exponential functions (represented as "Fits"), but the gross qualitative trends remain the same. 
Table S1. Structural parameters determined from the present study.

\begin{tabular}{c|cccccc}
\multicolumn{1}{c}{$M_{W}$} & $\phi(\%)^{\mathrm{b}}$ & $q^{*}\left(n m^{-1}\right)^{\mathrm{c}}$ & $d_{t}(n m)^{\mathrm{d}}$ & $I D(n m)^{\mathrm{e}}$ & $I D / 2 R_{g}{ }^{\mathrm{f}}$ & $\begin{array}{c}E_{\mathrm{a}}(q D=2)^{\mathrm{g}} \\
(\mathrm{kg} / \mathrm{mol})^{\mathrm{a}}\end{array}$ \\
\hline \multirow{2}{*}{9} & 6 & 0.203 & 31.0 & 7.4 & 1.42 & $149 \pm 5$ \\
& 16 & 0.215 & 29.2 & 5.6 & 1.09 & $117 \pm 5$ \\
& 27 & 0.245 & 25.6 & 2.0 & 0.38 & $123 \pm 14$ \\
\hline \multirow{2}{*}{38} & 6 & 0.223 & 28.1 & 10.0 & 0.92 & $145 \pm 3$ \\
& 16 & 0.299 & 21.0 & 2.8 & 0.26 & $93 \pm 40$ \\
& 27 & 0.338 & 18.6 & 0.4 & 0.04 & $20 \pm 10$ \\
\hline
\end{tabular}

${ }^{a} M_{\mathrm{W}}=\mathrm{P} 2 \mathrm{VP}$ molecular weight, ${ }^{\mathrm{b}} \phi=\mathrm{NP}$ volume fraction, ${ }^{\mathrm{c}} q^{*}=S(q)$ peak position, ${ }^{\mathrm{d}} d_{t}=\mathrm{NP}$ center-to-center spacing $\left(d_{t}=2 \pi / q^{*}\right),{ }^{\mathrm{e}} I D=$ face-to-face particle spacing $\left(\right.$ i.e., $\left.d_{t}-2 R_{N}\right),{ }^{\mathrm{f}} I D / 2 R_{\mathrm{g}}=$ confinement parameter. $R_{\mathrm{g}}=0.0277 M_{W}^{0.56}$ are calculated to be 2.6 and $5.4 \mathrm{~nm}$ for P2VP9k and P2VP38k, respectively, ${ }^{\mathrm{g}}$ Activation energy determined from the XPCS results at $q D=2$. The $E_{\text {a }}$ values remain nearly identical regardless of a choice of $q D$.

Table S2. Zero-shear viscosity $\left(\eta_{0}\right)$ of the P2VP bulk matrix determined from rheology.

\begin{tabular}{c|cc}
$M_{W}(\mathrm{~kg} / \mathrm{mol})$ & Temperature $\left({ }^{\circ} \mathrm{C}\right)$ & $\eta_{0}(\mathrm{~Pa} \mathrm{~s})$ \\
\hline \multirow{2}{*}{9} & 160 & 193 \\
& 170 & 87 \\
& 180 & 44 \\
\hline \multirow{2}{*}{38} & 160 & 7180 \\
& 170 & 2153 \\
\hline
\end{tabular}




\section{Theory Background and Additional Results}

\section{A. MCT and PRISM Theory Calculations}

For one-component fluids (here the spherical nanoparticle subsystem in a PNC), the well known MCT equation of motion for the (normalized) intermediate scattering function $F(q, t) \equiv$ $S(q, t) / S(q)$ is, ${ }^{7,8}$

$$
m \frac{d^{2} F(q, t)}{d t^{2}}+\frac{k_{\mathrm{B}} T q^{2}}{S(q)} F(q, t)+\int_{0}^{\infty} d s M_{\mathrm{tot}}(q, s) \frac{d F(q, t-s)}{d t}=0,
$$

The key physical quantity is the total memory function $M_{\text {tot }}$ which contains two parts: a short-time fast dynamics contribution that is $q$-independent and a delta function in time, and a long-time contribution approximated using the core idea of MCT to project collective stresses onto the bilinear product of collective density fields and factorize to the 2-point correlation level,

$$
\begin{aligned}
& M_{\mathrm{tot}}(q)=2 \zeta_{\mathrm{s}} \delta(t)+K_{\mathrm{MCT}}(q, t) \\
& K_{\mathrm{MCT}}(q)=\frac{\rho}{16 \pi^{3}} \int_{0}^{\infty} d \mathbf{k}\left|V_{\mathbf{k}, \mathbf{q}-\mathbf{k}}\right|^{2} F(k, t) F(|\mathbf{q}-\mathbf{k}|, t) .
\end{aligned}
$$

Here $m$ is the particle mass, $k_{\mathrm{B}}$ is the Boltzmann constant, $\rho$ is the particle number density, $\zeta_{\mathrm{s}}=$ $k_{\mathrm{B}} T / D_{\mathrm{s}}$ is the short-time friction constant, $\delta(t)$ is the Dirac delta function, and the "dynamic vertex" characterizing the wavevector dependent effective interparticle force and stress correlations is given by

$$
V_{\mathbf{k}, \mathbf{q}-\mathbf{k}}=(\widehat{\mathbf{q}} \cdot \mathbf{k}) C_{\mathrm{eff}}(k)+\widehat{\mathbf{q}} \cdot(\mathbf{q}-\mathbf{k}) C_{\mathrm{eff}}(|\mathbf{q}-\mathbf{k}|)
$$

with an effective direct correlation function playing the role of an effective interparticle pair potential:

$$
C_{\mathrm{eff}}(q)=\frac{1}{\rho}-\frac{1}{\rho S(q)}
$$


where $\mathbf{q}$ and $\mathbf{k}$ are wavevectors, $q=|\mathbf{q}|$ and $\widehat{\mathbf{q}}=\mathbf{q} /|\mathbf{q}|$.

The nanoparticle collective static structure factor, $S(q)$, for the silica-P2VP mixture is computed using PRISM integral equation theory of polymer nanocomposites as previously discussed. ${ }^{5}$ For a single effective interfacial attraction energy model parameterized for the P2VP38k system at $180^{\circ} \mathrm{C},{ }^{5}$ the results are shown in Figure S8 at different NP loadings. The corresponding real space pair correlation functions are shown in Figure S9a.

To compute the XPCS relaxation time, we take the overdamped limit and invoke the Markovian approximation in Eq. $(\mathrm{S} 1)$, i.e., $K_{\mathrm{MCT}}(q, t) \approx 2 K_{0}(q) \delta(t)$, corresponding to an exponential in time decay of the collective dynamic structure factor. Here $K_{0}(q)$ follows from performing the time integral of Eq. (S3) with a collective density fluctuation propagator approximated by its well-known short time/distance generalized diffusive form, $F(q, t)=$

$e^{-q 2 D_{s} t / S(q)}$, also known as the "cage diffusion" approximation in colloid physics. ${ }^{8,9}$ One then obtains $F(q, t)=e^{-t / \tau(q)}$ with the $q$-dependent collective relaxation time

$$
\tau(q)=\frac{S(q)}{q^{2} D_{\mathrm{s}}}\left(1+\frac{\rho}{16 \pi^{2}} \int_{0}^{\infty} d \mathbf{k} \frac{\left|V_{\mathbf{k}, \mathbf{q}-\mathbf{k}}\right|^{2}}{k^{2} / S(k)+(\mathbf{q}-\mathbf{k})^{2} / S(|\mathbf{q}-\mathbf{k}|)}\right)
$$

This is the central theoretical result in the main text, Eq. (6).

\section{B. Determination of Effective Contact Value and Short Time Friction}

The short-time diffusion constant $D_{\mathrm{s}}$ is also required to predict the $\phi$ dependence of $\tau(q)$ in Eq. (S6). As discussed in the main text, it is estimated as $D_{\mathrm{s}}=D_{0} / g\left(\phi_{\text {eff }}\right)$ in analogy with colloidal suspensions, ${ }^{10}$ where $D_{0}$ is the elementary NP diffusion coefficient related to the polymer 


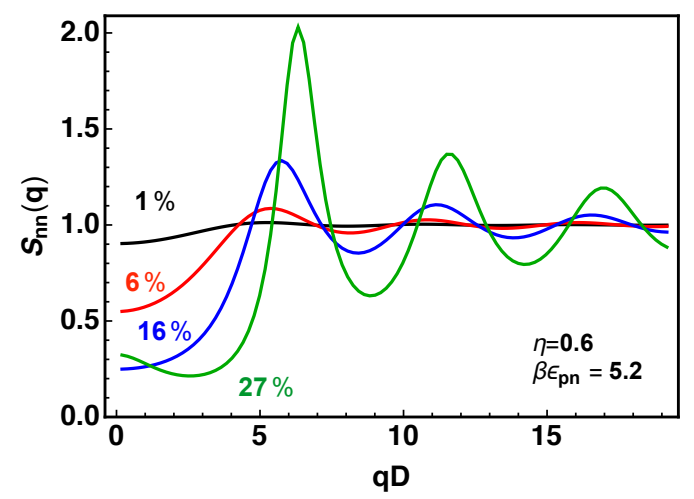

Figure S8. $S(q)$ of a single effective interfacial attraction energy PNC model obtained from PRISM theory.
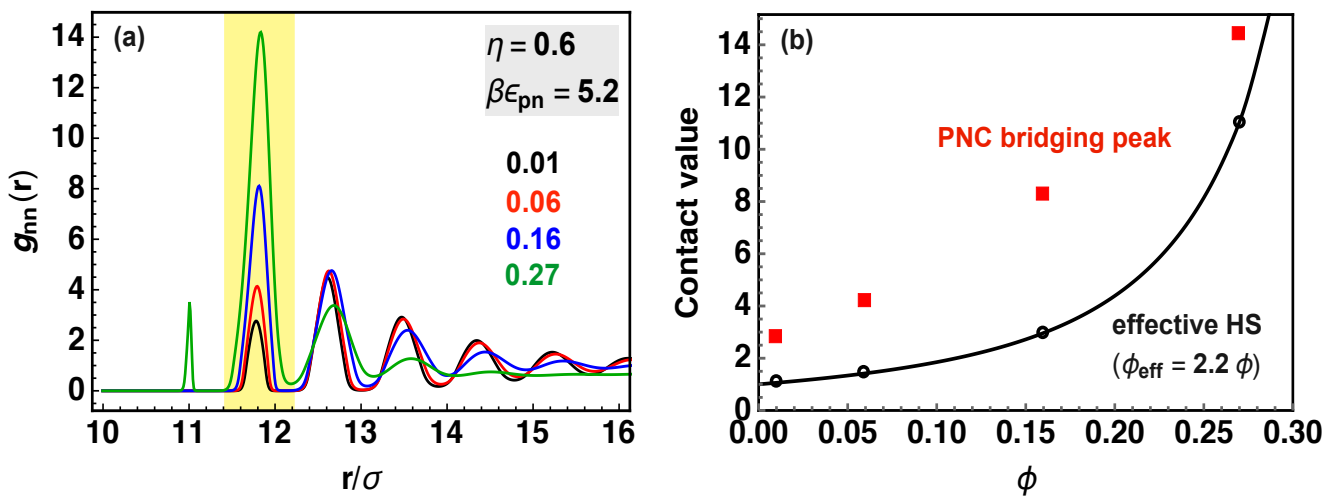

Figure S9. (a) PRISM theory NP-NP real space pair correlation function $g_{\mathrm{nn}}(r)$ at different NP loadings for parameters modeling the silica-P2VP PNC. The primary bridging peak is highlighted. (b) Contact value estimated from the PNC bridging peak predicted by PRISM theory and from a naive effective hard sphere model.

friction on a single NP, and $g\left(\phi_{\text {eff }}\right)$ is the contact value of an effective hard sphere fluid at an effective volume fraction $\phi_{\mathrm{eff}}(\phi)$. The latter is estimated by taking into account the interfacial polymer layer ${ }^{1-4}$ on the surface of nanoparticles of thickness $\delta$, and hence, $\phi_{\text {eff }}=\left(1+\delta / R_{N}\right)^{3} \phi$. Since this interfacial layer is insensitive to $M_{\mathrm{w}}{ }^{4}$ we choose $\delta=3 \mathrm{~nm}$ and $R_{N}=10 \mathrm{~nm}$ for both P2VP38k and P2VP9k systems based on SAXS experiments (Figure S2), which leads to $\phi_{\text {eff }} \approx$ $2.2 \phi$. The resulting contact values obtained using the highly accurate Ornstein-Zernike integral equations with modified Verlet closure for hard sphere (HS) fluids ${ }^{11}$ are shown in Figure S9b. We 
note that this approach is also consistent with a "dynamic" definition of layer thickness, in which the effective NP radius is determined by the distance of closest approach (classical turning point) between two nanoparticles that interact via an effective pair potential set by the theoretically predicted potential of mean force $\left(-k_{\mathrm{B}} T \ln g_{\mathrm{nn}}(r)\right.$, per Figure S9a) with an average collision energy of $1 k_{\mathrm{B}} T .^{5}$

One may alternatively estimate the contact value directly from the NP-NP pair correlation function, $g_{\mathrm{nn}}(r)$, for the PNC as predicted by PRISM theory. As seen in Figure S9a, the primary peak occurs at an inter-NP separation of $r=D+2 \sigma$ ( $D$ is the mean particle diameter), corresponding to a secondary tight bridging configuration. The value of this peak can be taken as an effective "contact value", which is compared with the effective HS model result in Figure S9b. Reassuringly, qualitative and often semi-quantitative agreement is found between these two distinct approaches, and (especially importantly) their dependence on $\phi$. But note that at $\phi=0$, the alternative estimate of a contact value exceeds unity, while the effective hard sphere model value is unity. Given we physically expect this contact value correction to the short-time diffusion vanishes in the dilute limit, we adopt the first method for our calculations in the main text. However, none of the qualitative behaviors and conclusions reported in the main text are influenced by which estimate of the contact value is employed.

\section{Effect of Strength of Polymer-Nanoparticle Attraction Energy}

The effect of reduction of the polymer segment adsorption energy (corresponding to weaker bridging or essentially no bridging) on the dynamical predictions in Figure 4 are shown in Figure S10. 


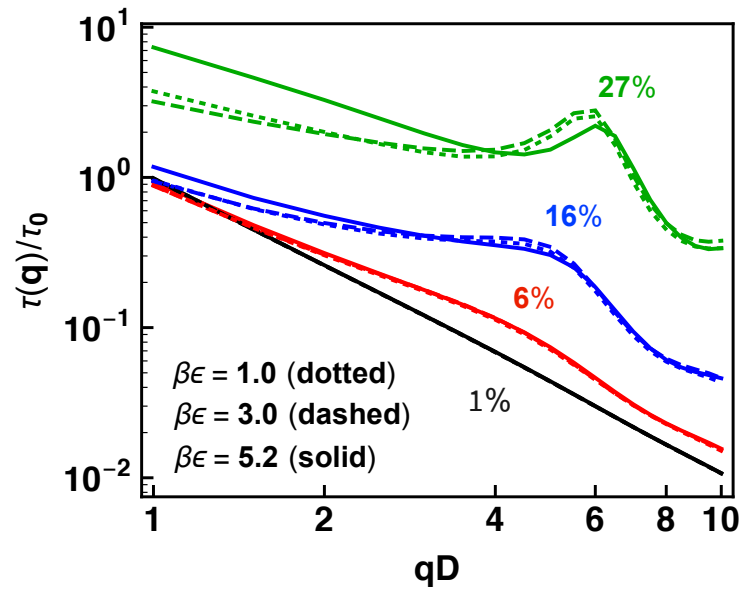

Figure S10. Dimensionless wavevector dependence of the non-dimensionalized MCT relaxation time $\tau(q)$ at different NP loadings and three values of interfacial attraction strength $\beta \epsilon_{\mathrm{pn}}$.

\section{Hard Sphere Suspension Model Predictions of the XPCS Relaxation Time}

The plots below underlie our brief comment in the text of the significant changes of the MCT predictions for the collective relaxation time based on adopting an effective 1-component hard sphere suspension model for all aspects of the dynamics of the nanoparticle subsystem in the PNC. The calculations follow the well-established ideas of Ref. 10 of the SI whence the short time
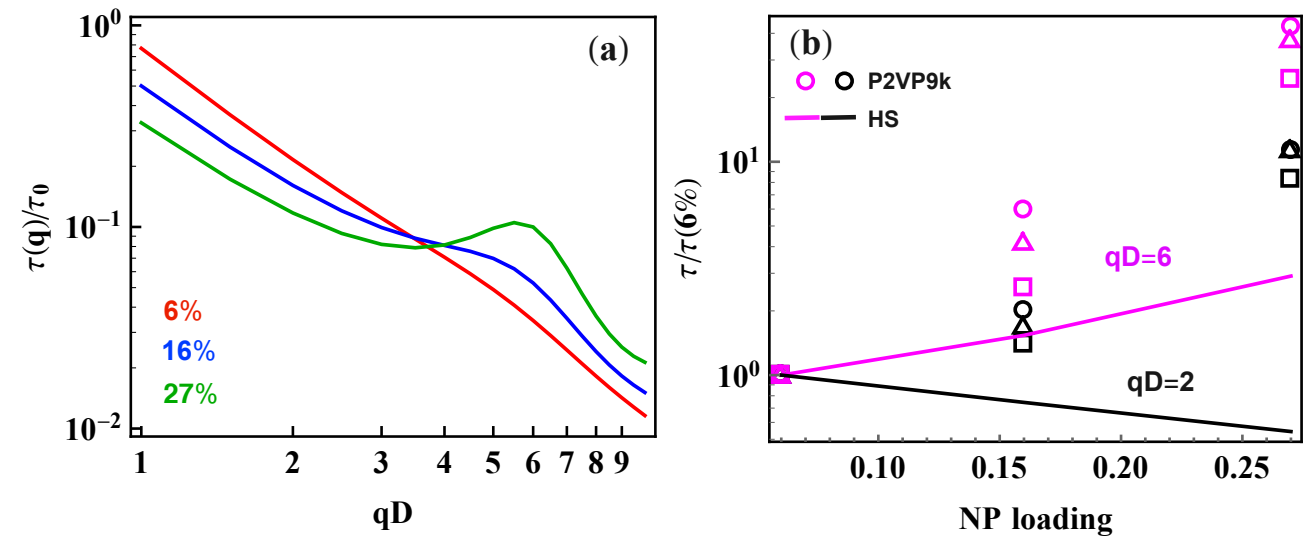

Figure S11. (a) $q$ and $\phi$ dependence of the non-dimensionalized MCT relaxation time $\tau(q)$ of a hard-sphere (HS) suspension. Panel (b) shows the experimental data for P2VP9k at $160{ }^{\circ} \mathrm{C}$ (squares), $170{ }^{\circ} \mathrm{C}$ (triangles), and $180{ }^{\circ} \mathrm{C}$ (circle) at $q D=2$ (black) and $q D=6$ (magenta) along with the predications based on the HS suspension model (indicated by the solid lines). 
friction constant is its hydrodynamic Stokes-Einstein value renormalized by binary collisions in a way that is known to be consistent with the experimental short time diffusion constant ${ }^{10}$ (and also an alternative 2-particle hydrodynamic analysis). The many body effects are evaluated with the same MCT approach employed in the main part of the article that relates structure to slower dynamics. The crucial difference from our theoretical analysis in the main text is the use of the literal hard sphere fluid pair structure $(S(q)$ and $g(r))$ instead of the proper PRISM theory derived forms for the PNC that include polymer-NP attractions and bridging. Both panels in Figure S11 show qualitative errors are incurred compared to experiment if one ignores how the polymer matrix re-organizes the NP subsystem structure.

\section{E. Effects of Polydispersity}

Incorporating size polydispersity (PD) in microscopic theories of structure (e.g., PRISM theory) and dynamics (e.g., MCT) is a challenging problem that is entirely open for complex PNC mixtures. Here we explore whether it is possible based on a simple practical averaging approximation to reproduce the cage peak intensity of the NP structure factor, $S_{\mathrm{nn}}\left(q^{*}\right)$, by choosing a sensible degree of PD consistent experiment. We find this is possible, which then allows us to analyze the consequences of PD on the dynamical theory predictions presented in the main text for a monodisperse NP model. We find even better agreement between experiment and theory, especially concerning the cage scale (high $q$ ) dependence of the collective relaxation time at high NP loadings.

\section{E.1. Polydispersity model and collective nanoparticle static structure factor}




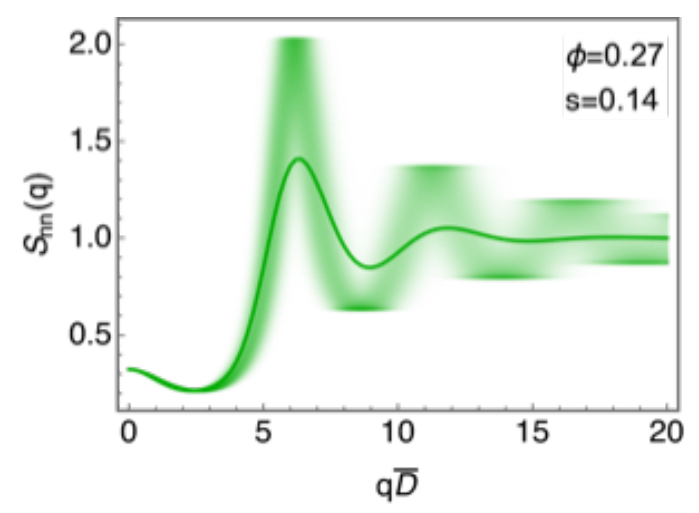

Figure S12. Example of polydispersity averaged $S_{n n}(q)$ at $27 \%$ loading and polydispersity index $s=0.14$ (solid line). $S_{n n}(q)$ of individual monodisperse systems in the distribution are also shown with opacity proportional to their size probability.

We consider the simplest approximation for the effect of NP size PD on $S_{\mathrm{nn}}(q)$ : PD is modeled via an ensemble of monodisperse systems with a proper particle size distribution in the spirit of an ideal mixing approximation. Since the breadth of the particle size distribution is relatively narrow (weak PD) for the experimental systems of interest, we ignore the indirect effect of NP-segment size ratio on $S_{\mathrm{nn}}(q)$. One then has that the size-normalized $S_{\mathrm{nn}}\left(q D_{i}\right)$ is identical to $S_{\mathrm{nn}}(q \bar{D})$ for all the monodisperse systems with diameter $D_{i}$ in the ensemble, where the latter is the structure factor is calculated using the standard PRISM theory of PNCs based on a monodisperse NP diameter $\bar{D}$. Hence, $S_{\mathrm{nn}}(q)=S_{\mathrm{nn}}\left(q \bar{D} / D_{i}\right)$, and the PD averaged static structure factor is:

$$
\left\langle S_{\mathrm{nn}}(q)\right\rangle=\int_{0}^{\infty} d D_{i} S_{\mathrm{nn}}\left(q \bar{D} / D_{i}\right) p\left(D_{i}\right)
$$

where $p\left(D_{i}\right)$ is the probability density function of a NP diameter. Based on prior experimental PNC studies, ${ }^{12}$ a log-normal distribution is adopted (we expect very similar results for a Gaussian or binomial distribution for the weak PD case of present interest), 


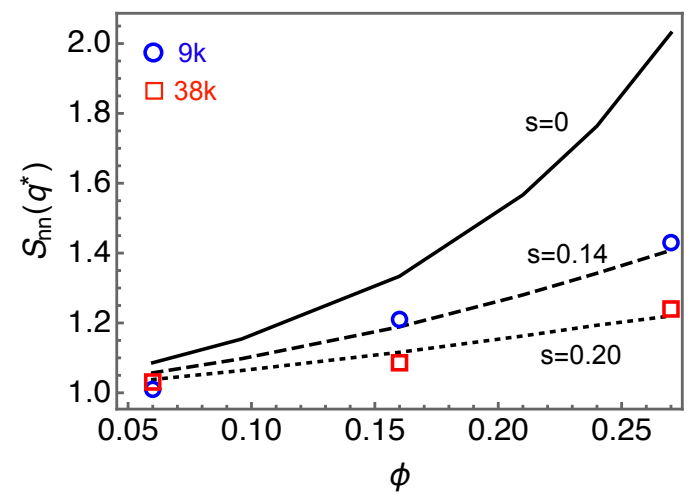

Figure S13. Cage peak intensity $S_{n n}\left(q^{*}\right)$ as a function of NP loading for different polydispersity indices of $s=0$ (monodisperse), 0.14 and 0.20 , compared to the experimental results for the P2VP9k and P2VP38k series.

$$
p(x ; \mu, \sigma)=\frac{1}{x \sigma \sqrt{2 \pi}} e^{\frac{-(\ln x-\mu)^{2}}{2 \sigma^{2}}}
$$

with the size PD variable $s$ defined as the fractional standard deviation $s=\sqrt{\exp \left[\sigma^{2}\right]-1}$ and the mean particle diameter $\bar{D}=\exp \left[\mu+\sigma^{2} / 2\right]$. From our SAXS measurements, we find $s=0.17$ and 0.19 for the $\bar{D}=23.6 \mathrm{~nm}$ and $18.2 \mathrm{~nm}$ diameter P2VP9k and P2VP38k samples, respectively. Figure S12 shows a representative example of the result of such an averaging procedure for $S_{\mathrm{nn}}(q)$ at $27 \%$ loading with $s=0.14$.

We expect the most PD-sensitive feature is the interparticle cage peak intensity $S_{\mathrm{nn}}\left(q^{*}\right)$, and adopt it as the metric for determining a single PD index that best reproduces the experimental $S_{\mathrm{nn}}\left(q^{*}\right)$ for all loadings of a given PNC system. The results in Figure S13 show that $s=0.14$ and 0.2 captures very well the $S_{\mathrm{nn}}\left(q^{*}\right)$ data for the P2VP9k and P2VP38k systems, respectively. Moreover, the absolute value of $s$ and its system dependence are remarkably consistent with the experimental values. This suggests the softening of the cage peak in experiment at high $M_{\mathrm{w}}$ is mainly due to a larger PD, as we hypothesized in our prior work. ${ }^{5}$ 

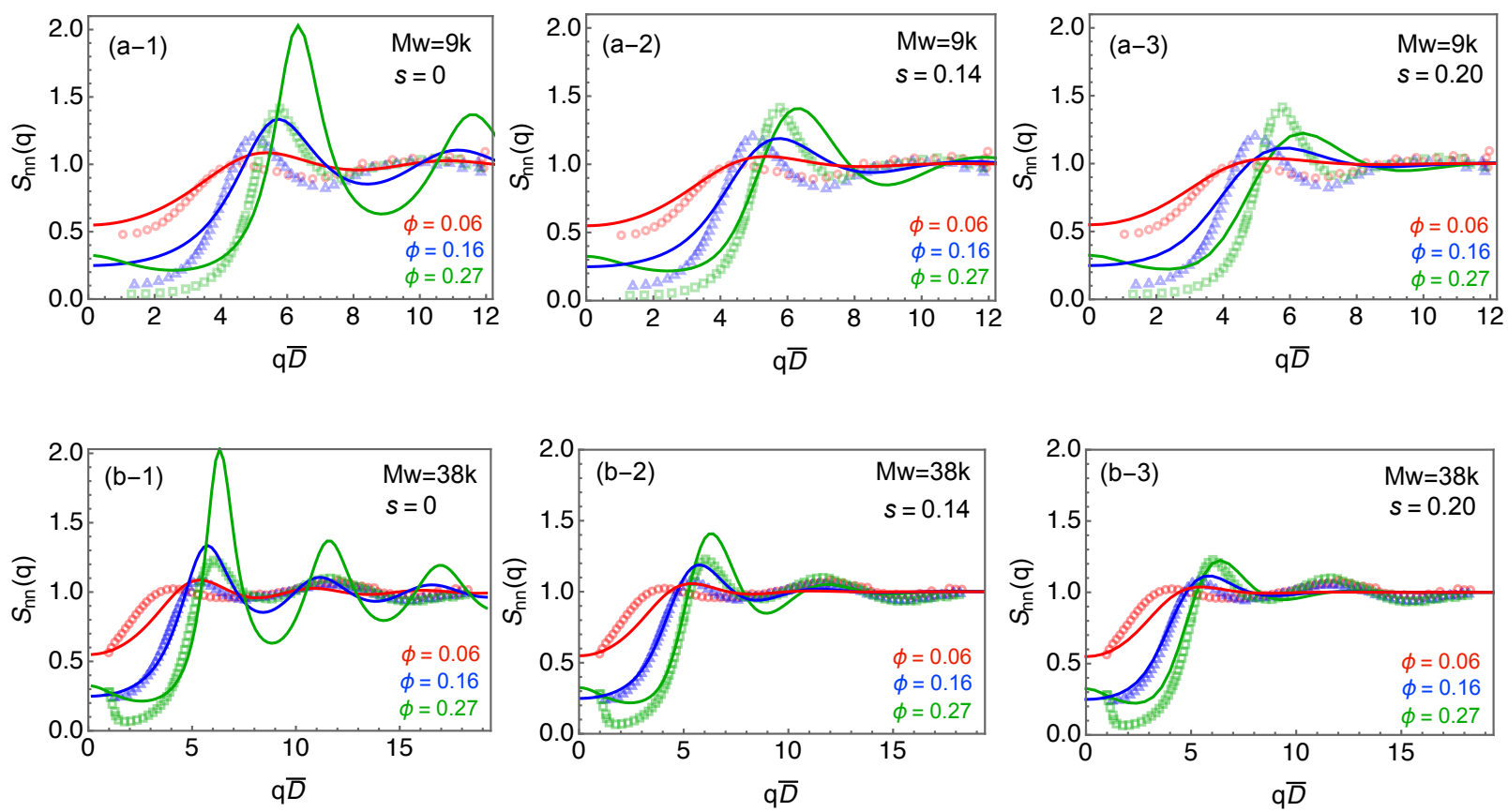

Figure S14. Polydispersity-corrected $S_{n n}(q)$ for $s=0$ (monodisperse), 0.14, and 0.2 compared to the experimental data for the P2VP9k and P2VP38k series at the different NP loadings.

To see how PD affects $S_{\mathrm{nn}}(q)$ at different wavevectors, we overlay in Figure S14 the theoretical and experimental $S_{\mathrm{nn}}(q)$. Clearly PD has a weaker effect in the low- $q$ regime than the high- $q$ regime. Importantly, the aspect of $S_{\mathrm{nn}}(q)$ that is of most importance in the dynamic MCT is the high- $q$ structural correlations. Overall, we find the theory-experiment agreement for $S_{\mathrm{nn}}(q)$ is much improved with $s=0.14$ and 0.2 for the P2VP9k and P2VP38k series, respectively, compared to the monodisperse $(s=0)$ results also shown in Figure S14. Of course, exact reproduction is challenging, but the cage peak is especially sensitive to PD and we can accurately capture it without affecting much $S_{\mathrm{nn}}(q=0)$ which was used for the model calibration (total packing fraction, interfacial attraction) here and in our previous analysis. ${ }^{5}$ The better agreement for the P2VP38k system compared to the P2VP9k PNC is expected since our model parameters were previously calibrated based on the P2VP38k system. ${ }^{5}$ 


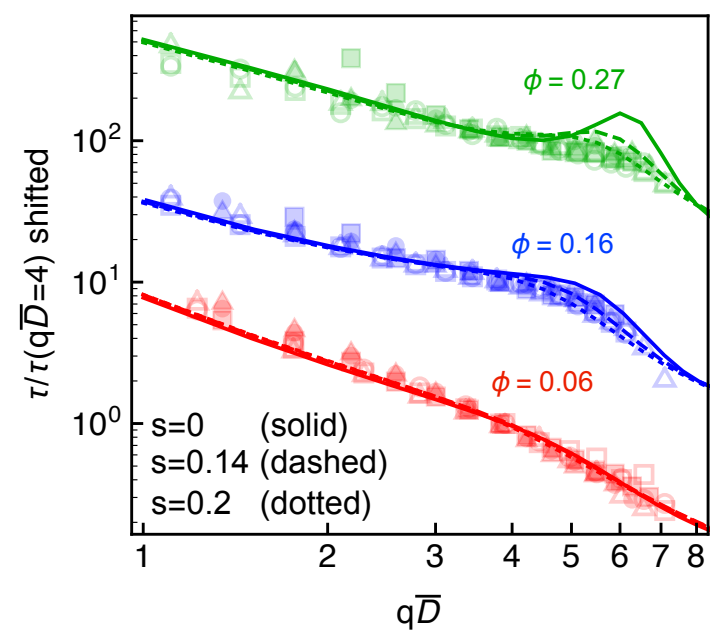

Figure S15. Normalized polydispersity-corrected $\tau(q)$ with $s=0$ (monodisperse), 0.14 and 0.2 compared to the experimental results (indicated by symbols) for the P2VP9k and P2VP38k at the three temperatures and different NP loadings shown in Figure 4.

\section{E.2. q-dependent collective NP dynamics}

We now explore the effect of PD on collective NP dynamics. The dynamic observable to be PD-averaged should be the time correlation function, $F(q, t)=e^{-t / \tau(q)}$, now given by

$$
\langle F(q, t)\rangle=\int_{0}^{\infty} d D_{i} e^{-t / \tau\left(q \bar{D} / D_{i}\right)} p\left(D_{i}\right) \approx e^{-\langle 1 / \tau(q)\rangle t}
$$

The final approximate equality follows from a first order cumulant expansion which is justified since PD is weak, and most experimental time correlation functions decay exponentially. The latter is the same simplification invoked above for the monodisperse model (Markov limit). The resulting PD-corrected relaxation time is

$$
\bar{\tau}(q)=1 /\langle 1 / \tau(q)\rangle=1 / \int_{0}^{\infty} d D_{i} \frac{1}{\tau\left(q \bar{D} / D_{i}\right)} p\left(D_{i}\right)
$$

Figure S15 shows the effect of PD on $\tau(q)$ for $s=0$ (monodisperse), 0.14 , and 0.2 , compared to the experimental master curve of the silica-P2VP systems with P2VP9k and P2VP38k 

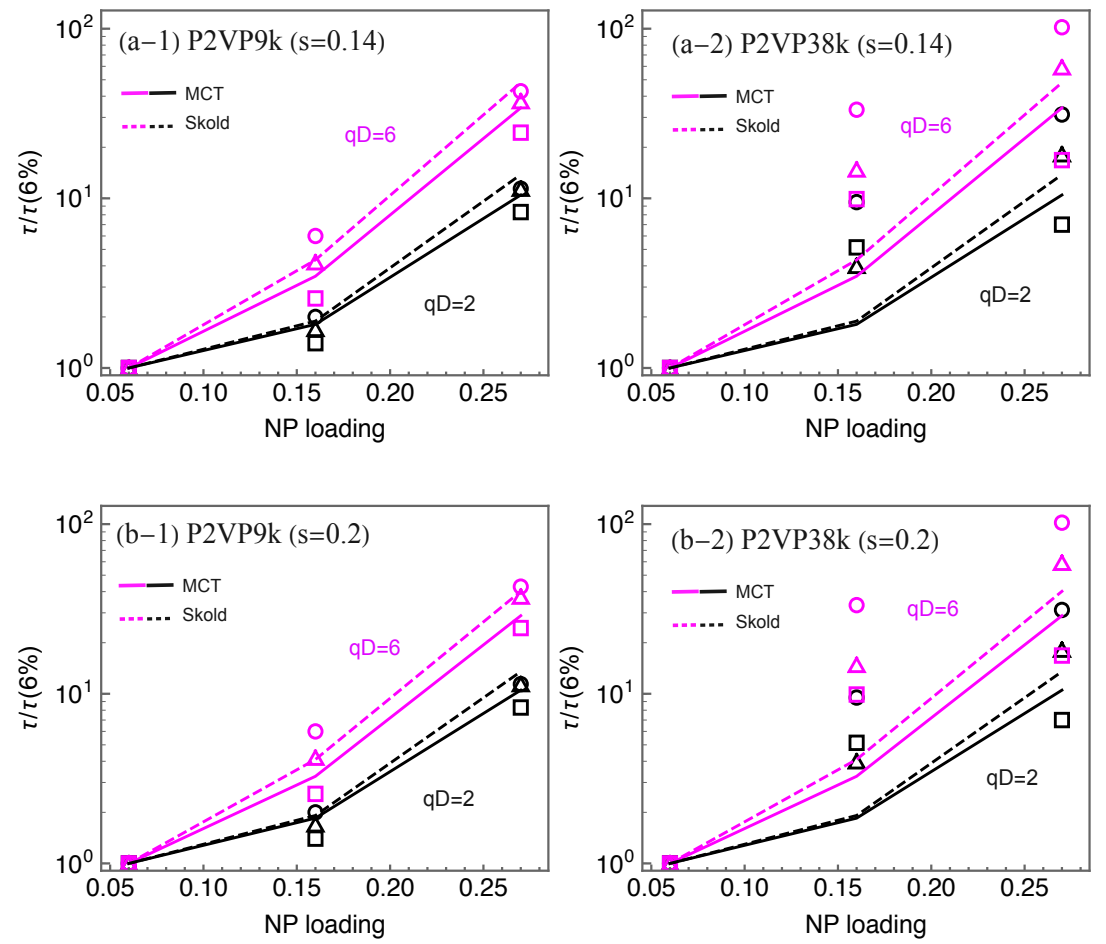

Figure S16. Normalized polydispersity-corrected $\tau$ at $q D=2$ and 6 as a function of NP loading with (a) $s=0.14$ and (b) $s=0.2$. The experimental values for the P2VP9k and P2VP38k series are shown for comparison. See Figure 6 of the main article for the analogous $s=0$ monodisperse model results.

and three temperatures as discussed in the main text (Figure 4). We find for the same optimal PD value used for $S_{\mathrm{nn}}(q)$, the cage scale peak in $\tau(q)$ at $27 \%$ loading is now almost entirely suppressed, consistent with experiment. Moreover, changes of the $q$-dependence of the relaxation time are virtually negligible at other wavevectors for $27 \%$ loading, and for almost all wavevectors for $6 \%$ and $16 \%$ loadings. These results strongly buttress the conclusions drawn in the main text concerning Figure 4 based on the monodisperse NP model. They are also of intrinsic interest in that they suggest weak PD is apparently a second order effect except at high enough loadings that the NP structural cage peak is of significant amplitude.

We applied the same procedure to obtain the PD-averaged Skold approximation $\tau(q)$ discussed in the main text (and see Figure 4) and find similar trends of suppressed cage peak as shown for the MCT-based analysis. But the Skold approximation results remain clearly inferior to 
the MCT counterpart as established in the main text based on the monodisperse model, and as expected physically.

Figure S16 shows the normalized $\phi$ dependence of the collective relaxation time compared to the experimental data at the same two reduced wavevectors studied in the main text. Comparing these results for $s=0.14$ and 0.20 with their analogs for $s=0$ in Figure 6, one sees that the overall relative relation between the predictions of MCT and its Skold approximation analog and our experimental data remain essentially identical to what was presented in the main text for the monodisperse model.

\section{References}

1. Cheng, S. W.; Holt, A. P.; Wang, H. Q.; Fan, F.; Bocharova, V.; Martin, H.; Etampawala, T.;White, B. T.; Saito, T.; Kang, N. G.; Dadmun, M. D.; Mays, J. W. ; Sokolov, A. P. Unexpected Molecular Weight Effect in Polymer Nanocomposites. Phys. Rev. Lett. 2016, 116, 5.

2. Genix, A. C.; Bocharova, V.; Kisliuk, A.; Carrol, B.; Zhao, S.; Oberdisse, J. ; Sokolov, A. P. Enhancing the Mechanical Properties of Glassy Nanocomposites by Tuning Polymer Molecular Weight. ACS Appl. Mater. Interfaces 2018, 10, 33601-33610.

3. Popov, I.; Carroll, B.; Bocharova, V.; Genix, A.-C.; Cheng, S.; Khamzin, A.; Kisliuk, A. ; Sokolov, A. P. Strong Reduction in Amplitude of the Interfacial Segmental Dynamics in Polymer Nanocomposites. Macromolecules 2020, 53, 4126-4135.

4. Holt, A. P.; Bocharova, V.; Cheng, S.; Kisliuk, A. M.; White, B. T.; Saito, T.; Uhrig, D.; Mahalik, J. P.; Kumar, R.; Imel, A. E.; Etampawala, T.; Martin, H.; Sikes, N.; Sumpter, B. G.; Dadmun, M. D. ; Sokolov, A. P. Controlling Interfacial Dynamics: Covalent Bonding versus Physical Adsorption in Polymer Nanocomposites. ACS Nano 2016, 10, 6843-6852.

5. Zhou, Y.; Yavitt, B.; Zhou, Z.; Bocharova, V. ; Salatto, D. ; Endoh, M. ; Ribbe, A. ; Sokolov, A. ; Koga, T. ; Schweizer, K. S. Bridging Controlled Network Microstructure and Long Wavelength Fluctuations in Silica-Poly(2-Vinylpyridine) Nanocomposites: Experimental Results and Theoretical Analysis. Macromolecules 2020, 53, 6984-6994.

6. Griffin, P. J.; Bocharova, V.; Middleton, L. R.; Composto, R. J.; Clarke, N.; Schweizer, K. S. ; Winey, K. I. Influence of the Bound Polymer Layer on Nanoparticle Diffusion in Polymer Melts. ACS Macro Lett. 2016, 5, 1141-1145.

7. Reichman, D. R. ; Charbonneau, P. Mode-Coupling Theory. J. Stat. Mech. Theory Exp. 2005, 2005, P05013

8. Hansen, J.-P. P.; McDonald, I. R. Theory of Simple Liquids; Elsevier, Burlington, 2006.

9. Hess, W.; Klein, R. Generalized Hydrodynamics of Systems of Brownian Particles. Adv. Phys. 1983, 32, 173-283. 
10. Cohen, E. G. D.; Verberg, R.; Schepper, I. M. de. Viscosity and Diffusion in Hard-SphereLike Colloidal Suspensions. Physica A 1998, 251, 251-265.

11. Zhou, Y.; Mei, B.; Schweizer, K. S. Integral Equation Theory of Thermodynamics, Pair Structure, and Growing Static Length Scale in Metastable Hard Sphere and Weeks-ChandlerAndersen Fluids. Phys. Rev. E. 2020, 101, 42121.

12. Gam, S.; Meth, J. S.; Zane, S. G.; Chi, C.; Wood, B. A.; Winey, K. I.; Clarke, N.; Composto, R. J. Polymer Diffusion in a Polymer Nanocomposite: Effect of Nanoparticle Size and Polydispersity. Soft Matter 2012, 8, 6512-6520. 